Documents pour l'histoire des techniques

Nouvelle série

$17 \mid 1^{\text {er }}$ semestre 2009

L'invention technique et les figures de l'inventeur (XVIII $-X^{\mathrm{e}}$ siècles)

\title{
Fabrication de l'acide sulfurique et des engrais chimiques (1887) : quand Ernest Sorel (1850-1904) faisait du « génie des réacteurs »...
}

Fabrication de l'acide sulfurique et des engrais chimiques (1887) : when Ernest Sorel (1850-1904) acted as a chemical reaction engineer...

\section{OpenEdition}

Journals

Édition électronique

URL : http://journals.openedition.org/dht/559

DOI : $10.4000 /$ dht.559

ISSN : 1775-4194

Éditeur :

Centre d'histoire des techniques et de l'environnement du Cnam (CDHTE-Cnam), Société des élèves du CDHTE-Cnam

\section{Édition imprimée}

Date de publication : 31 mars 2009

Pagination : 209-218

ISBN : 978-2-95-30779-3-3

ISSN : 0417-8726

\section{Référence électronique}

Jacques Breysse, «Fabrication de l'acide sulfurique et des engrais chimiques (1887) : quand Ernest Sorel (1850-1904) faisait du " génie des réacteurs »... », Documents pour l'histoire des techniques [En

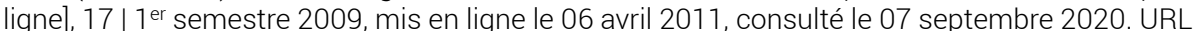
http://journals.openedition.org/dht/559 ; DOI : https://doi.org/10.4000/dht.559 


\title{
Fabrication de l'acide sulfurique et des engrais chimiques (1887) : quand Ernest Sorel (1850-1904) faisait du "génie des réacteurs "...
}

\author{
Jacques Breysse \\ CDHTE-Cnam
}

\begin{abstract}
Résumé
Ernest Sorel est internationalement connu pour ses travaux en distillation/rectification. II l'est beaucoup moins pour ses contributions à l'étude et à l'amélioration des grands procédés industriels en chimie minérale et plus particulièrement à celui de la fabrication de l'acide sulfurique par le procédé des chambres de plomb. II publie pourtant en 1887 un livre extrêmement complet sur ce dernier sujet. Cet ouvrage présente beaucoup d'éléments atypiques par comparaison avec des ouvrages de chimie industrielle de la même époque, comme par exemple en introduction de très nombreux tableaux de constantes physiques et autres données de base, ou encore diverses parties sur la manière de " conduire " une unité. Mais surtout, à plusieurs reprises il essaie de "modéliser » les phénomènes physico-chimiques rencontrés sous forme d'équations mathématiques, caractéristique d'une « approche modélisation » tout à fait moderne. C'est ainsi qu'il essaie de modéliser la transformation chimique tout au long des chambres de plomb avec l'aide d'une équation cinétique équivalente à une vitesse de réaction du pseudo premier ordre associé à un fonctionnement de type " piston », approche qui ne sera formalisée que bien plus tard, à partir des années 1930. Sorel, déjà grand précurseur dans le domaine de la distillation continue, apparaît ici comme un des pionniers du « génie des réacteurs ».
\end{abstract}

Résumés et mots clés en anglais sont regroupés en fin de volume, accompagnés des mots clés français

$\mathrm{E}^{\mathrm{m}}$ nest Sorel est internationalement connu pour ses travaux dansle domaine de la distillation', domaine dans lequel il a acquis une notoriété comme pionnier, célébré par ses pairs². II est par contre beaucoup

1 Ernest Sorel (1850-1904) est diplômé de l'École polytechnique (Corps des manufactures de l'État) en 1871. Après un long passage dans l'industrie chimique (Malétra, SaintGobain), il se spécialise en distillation et en particulier dans celle de l'alcool. II devient directeur technique de l'entreprise Savalle, fabricant de colonnes à distiller, au début des années 1890 avant de se consacrer à l'enseignement et à des missions d'expert au tournant du siècle et jusqu'à sa mort en 1904. Sorel est l'auteur de nombreux articles en distillation/rectification et de trois ouvrages : La rectification de l'alcool, Encyclopédie des aide-mémoires, Paris, Gauthier-Villars/Masson, 1893 ; La distillation, Encyclopédie des aide-mémoires, Paris, Gauthier-Villars/Masson, 1895 ; Distillation et rectification industrielles, Paris, Carré et Naud, 1899. 2 William H. Walker Warren K. Lewis \& William H. McAdams, auteurs en 1923 d'un des tout premiers ouvrages aux États- moins connu pour ses contributions à l'étude et à l'amélioration des grands procédés industriels de chimie minérale et plus particulièrement à celui de la fabrication de l'acide sulfurique. II publie pourtant en 1887 un ouvrage extrêmement complet concernant

Unis d'ingénierie chimique, Principles of chemical engineering, McGraw-Hill, 1923, soulignent ainsi son rôle : " La théorie mathématique des colonnes de rectification dans le cas de mélanges binaires, a été définie pour la première fois par Ernest Sorel, qui a calculé l'enrichissement progressif plateau par plateau au moyen de bilans matières et énergie entrée/ sortie de chaque étage et en considérant que l'équilibre thermodynamique était réalisé entre les vapeurs et le liquide " (" The mathematical theory of the rectifying column as applied to the distillation of binary mixtures was first developed by Ernest Sorel, who calculated the enrichment from plate to plate by equating the amount of energy and of matter entering and leaving each plate and by assuming that equilibrium was realized between the vapors and the liquid through which it bubbled ॥) ( p. 594). 


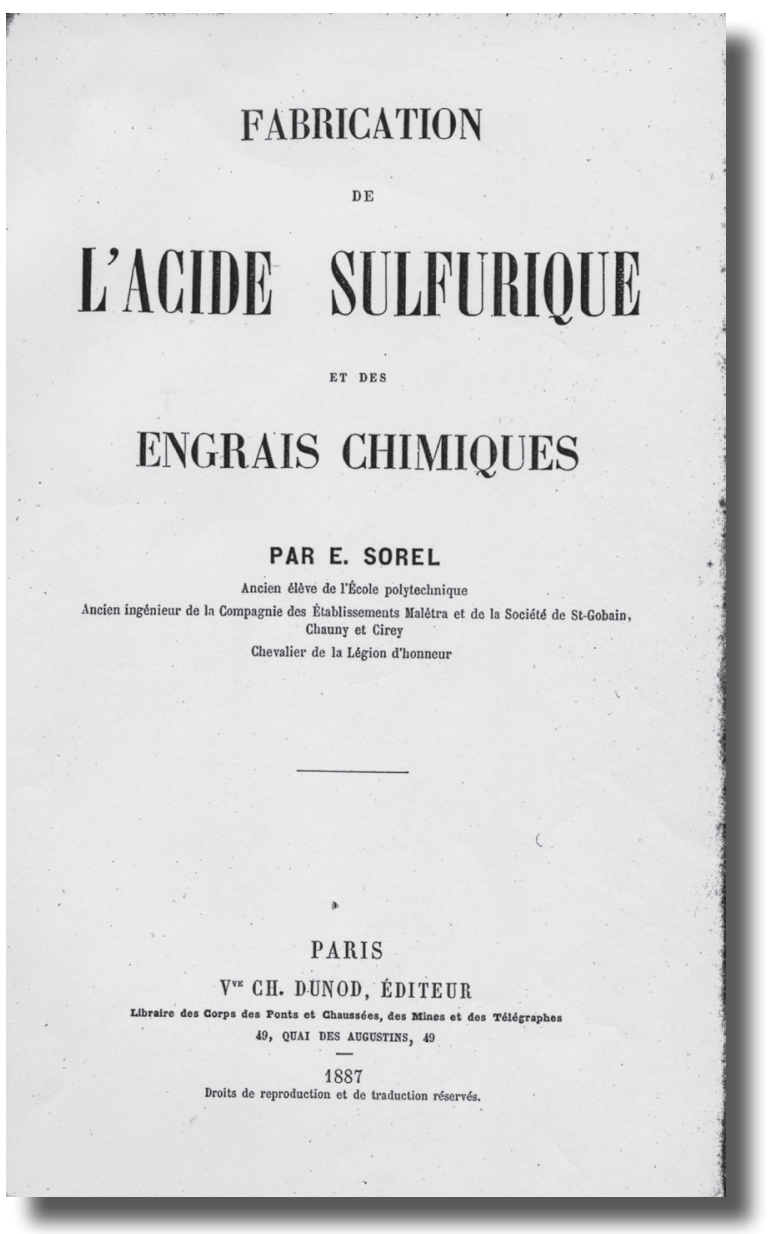

la fabrication de l'acide sulfurique ${ }^{3}$, Fabrication de l'acide sulfurique et des engrais chimiques, après avoir pris un brevet l'année précédente sur l'amélioration du procédé4. II s'appuie alors sur sa riche expérience industrielle, d'abord chez Malétra de 1873 à 1882 (Roven et Saint Denis) puis chez Saint-Gobain de 1882 à 1886 (Usine de l'Oseraie dans le Vaucluse). II reprendra aussi certains éléments de cet ouvrage dans les années 1890 à travers des articles publiés dans des grandes revues scientifiques ${ }^{5}$.

3 Ernest.Sorel, Fabrication de l'acide sulfurique et des engrais chimiques, Paris, Dunod, 1887. L'ouvrage comporte en introduction de très nombreuses tables de données de base (75 pages), puis aborde successivement la structure chimique des composés en causes, les problèmes de matières premières et la description détaillée des différents procédés. C'est au cours de ces chapitres qu'il évoque les aspects cinétiques, la manière de conduire les unités, ainsi que le modèle chimique de la réaction. Cet ouvrage est difficile à trouver. Il n'existe pas à la Bibliothèque Nationale de France, et à ce jour nous n'avons pas trouvé de bibliothèque académique en France qui en disposait. Nous ne connaissons que deux exemplaires appartenant à des personnes privées.

4 Brevet n 179203 (Août 1886), « Perfectionnement à la fabrication de l'acide sulfurique $॥$.

5 Bulletin de la Société industrielle de Mulhouse, 1889, pp.
Ses travaux dans le domaine ne sont pas toujours reconnus à leur juste valeur, mais on peut cependant mentionner ce qu'écrivait en 1891 Georges Lunge - le grand spécialiste du domaine au XIXe siècle ${ }^{6}$ - dans la Revue générale des sciences pures et appliquées : "Les perfectionnements qui ont été proposés de divers côtés, en particulier par l'auteur [Lunge], doivent encore subir la sanction d'une plus longue expérience. II suffira donc de rappeler que ces propositions ont été fondées sur une nouvelle théorie de la formation de l'acide sulfurique, théorie qui a d'abord été développée par l'auteur, et peu après d'une façon indépendante par M. Sorel ${ }^{7}{ }^{7}$, et ce que, bien plus tard, dira Paul Baud en 1951 dans son ouvrage de chimie industrielle ${ }^{8}$ : "On admet le plus souvent que le rôle des composés oxygénés de l'azote dans la formation de l'acide sulfurique est régi sensiblement par la série de réactions clairement formulées par E. Sorel ... ॥.

Toutefois, c'est dans le domaine de la cinétique de réaction que nous voudrions présenter la contribution d'Ernest Sorel. II va en effet développer dans le cas de l'acide sulfurique, une approche tout à fait nouvelle et originale pour l'analyse et la conduite de I'installation, approche qui préfigure étonnamment un domaine, le "génie des réacteurs », qui n'émergera formellement que cinquante ans plus tard, à la fin des années $1930^{\circ}$.

240-293, Revue générale des sciences pures et appliquées en 1893, vol. 4, pp. 350-353, et 1895, vol. 6, pp. 839-846, Bulletin de la société d'encouragement en 1895, pp.138-147.

6 Georges Lunge publia en 1879 (1ère éd.) (avec J. Naville) un ouvrage de référence, le Traité de la fabrication de la soude, en deux volumes, dont le ler volume était intégralement consacré à la fabrication de l'acide sulfurique. Cet ouvrage était une traduction en français d'un ouvrage du même auteur qui venait d'être publié en Allemagne.

7 Georges Lunge, "La grande industrie chimique ", Revue générale des sciences pures et appliquées, $n^{\circ} 2,1891$, pp. 39-47. Lunge faisait allusion à trois de ses articles dans Berichte (1885 et 1888) et à l'article de Sorel de 1889 dans le Bulletin de la Société industrielle de Mulhouse de 1889, mais sans mentionner le brevet que prit Sorel sur ce sujet dès 1886 , ni surtout son ouvrage de 1887.

8 Paul Baud, Traité de chimie industrielle, $44^{\mathrm{èm}}$ éd., Paris, Masson, 1951, tome 1, p. 39.

9 Le « Génie des réacteurs (GR) » ou encore « Génie de la réaction chimique (GRC) ॥ (Chemical Reaction Engineering) est une des disciplines majeures du "génie des procédés ". Elle concerne l'étude et la modélisation du fonctionnement des réacteurs chimiques. Elle intègre, entre autres, des éléments de thermodynamique, de cinétique chimique, de mécanique des fluides et des lois "locales " de transport (transferts de matières, de chaleur et de quantités de mou- 
Le procédé en cause est celui dit des « chambres de plomb $\| 10$. On réalise l'oxydation par l'air en phase gazeuse de l'anhydride sulfureux, en présence de vapeur d'eau et de vapeurs nitreuses, à une température voisine de $50^{\circ} \mathrm{C}$. Ceci est réalisé dans d'immenses "chambres de plomb " ou l'acide sulfurique est produit en solution aqueuse sous forme de gouttelettes " en suspension " et ruisselantes sur les parois. Un des problèmes clé de la conduite du procédé concerne la maîtrise de la température qui ne doit pas être trop élevée pour ne pas favoriser la solubilisation des vapeurs nitreuses dans les solutions d'acide sulfurique produit (voir planche jointe).

Sorel, dans un paragraphe intitulé « Sur la vitesse de transformation de l'acide sulfureux en acide sulfurique " de son ouvrage de 1887 (pp. 358-365), examine dans le détail les aspects cinétiques et thermiques du procédé (on trouvera un fac-similé des pages concernées à la fin de cet article).

Sorel introduit le sujet comme suit :

«Si, dans chaque section transversale de la chambre, étaient réunies toutes les conditions les plus favorables à la transformation de l'acide sulfureux en acide sulfurique, il est clair que la vitesse de transformation serait proportionnelle presque

vement). On admet en général que les premiers travaux qui préfigurent les bases de la discipline sont ceux de Olaf Hougen et Kenneth Watson aux Ełats-Unis (Olaf A. Hougen et Kenneth M. Watson, Industrial chemical calculation, New York, Wiley, 1936) et de Gerhard Damköhler en Allemagne (Gerhard Damköhler, " Einfluss von diffusion, strömung und wärmetransport auf die ausbeute bei chemisch-technischen reaktionen ॥, dans Arnold Eucken et Max Jakob éd., Der chemie-ingenieur, Leipzig, Akad. Verlagsgesellshaft, 1937, Vol. 3, Partie 1, pp. 359-485) à la fin des années 1930. Mais c'est surtout après guerre que la discipline s'est développée avec notamment les ouvrages généraux de Octave Levenspiel aux États-Unis (Octave Levenspiel, Chemical reaction engineering, New York, Wiley, 1972) et de Jacques Villermaux en France (Jacques Villermaux, Génie de la réaction chimique, Paris, Tech \& Doc, 1982).

10 Concernant l'histoire de la fabrication de l'acide sulfurique, on pourra se reporter à l'article très général de André Thépot, L'Usine nouvelle, "L'acide sulfurique ", octobre 1979, pp. 200-211, et aux ouvrages de John G. Smith, Heavy chemical industry in France. The origins and development in France, Clarendon Press, Oxford, 1979 et de John R. Harris, Industrial Espionage and technology tranfer, Britain and France in the XVIII th century, Aldershot, Ashgate, 1998 concernant l'émergence du procédé et son développement en France. Sur la description détaillée du procédé on pourra se reporter par exemple à l'ouvrage de Paul Baud, op. cit. note 8, pp. 19-95. exactement au taux d'acide sulfureux restant dans le mélange qui traverse cette tranche $»$.

"l écrit à la suite l'équation"l :

$-d \sigma / d l=K . \sigma$

Après intégration, il aboutit à une fonction puissance qui lui permet de représenter l'évolution du taux d'acide sulfureux en fonction de la distance I dans la chambre :

$\sigma / 100=(\varphi / 100)^{1 / L}$

où $\sigma$ représente le taux d'acide sulfureux restant à la distance I et $\varphi$ le taux d'acide sulfureux restant à la distance $\mathrm{L}^{12}$.

Sorel considère que la réaction a lieu dans les chambres sous la forme d'un front qui avance sans se re-mélanger. Même si cette « modélisation » paraît à la limite simpliste ${ }^{13}$, force est de constater que la

$11 \sigma$ taux centésimal d'acide sulfureux dans une "tranche " d'épaisseur dl, distante de I de la tête de la chambre do variation de $\sigma$ entre l'entrée et la sortie de la «tranche »; K constante.

12 Approche «génie des réacteurs » actuelle :

En considérant que dl épaisseur de la tranche $=d V / S$

(S aire de la section droite de la " chambre ", dV volume de la tranche), on obtient:

- $d \sigma / d V=(K / S) . \sigma$

en assimilant $\sigma$ et $C$, cette équation correspond presque formellement à celle que l'on obtient avec ce que l'on appelle aujourd'hui un réacteur "piston "), c'est-à-dire un réacteur " ouvert" (continu) ou la réaction se propage sous forme d'un front qui avance sans se re-mélanger avec ce qu'il y a devant ou derrière. Dans le cas d'une réaction chimique très simple $r=k C$

( $r$ vitesse de la réaction, $k$ constante de vitesse, C concentration en réactif), la transformation chimique dans un réacteur de type « piston » s'écrit :

$-d C / d V=r /(Q O . C O)=[k /(Q O . C O)] . C$

$\mathrm{dC}$ variation de concentration dans un élément de volume $\mathrm{dV}$ Qo débit d'entrée dans le réacteur

Co concentration en réactif à l'entrée du réacteur.

Cette équation est équivalente à celle de Sorel à la seule différence que Sorel n'explicite pas K en prenant en compte l'effet du débit d'entrée Qo.

Après intégration on obtient :

$C=C O \operatorname{Exp}\{-K . \vee / Q O\}$

caractéristique d'une décroissance exponentielle de la concentration en réactif entre l'entrée et la sortie du réacteur de volume $\mathrm{V}$.

13 A priori on aurait pu penser que chaque chambre aurait 
DISPOSITION D'UNE FABRIQUE D'ACIDE SULFURIQUE
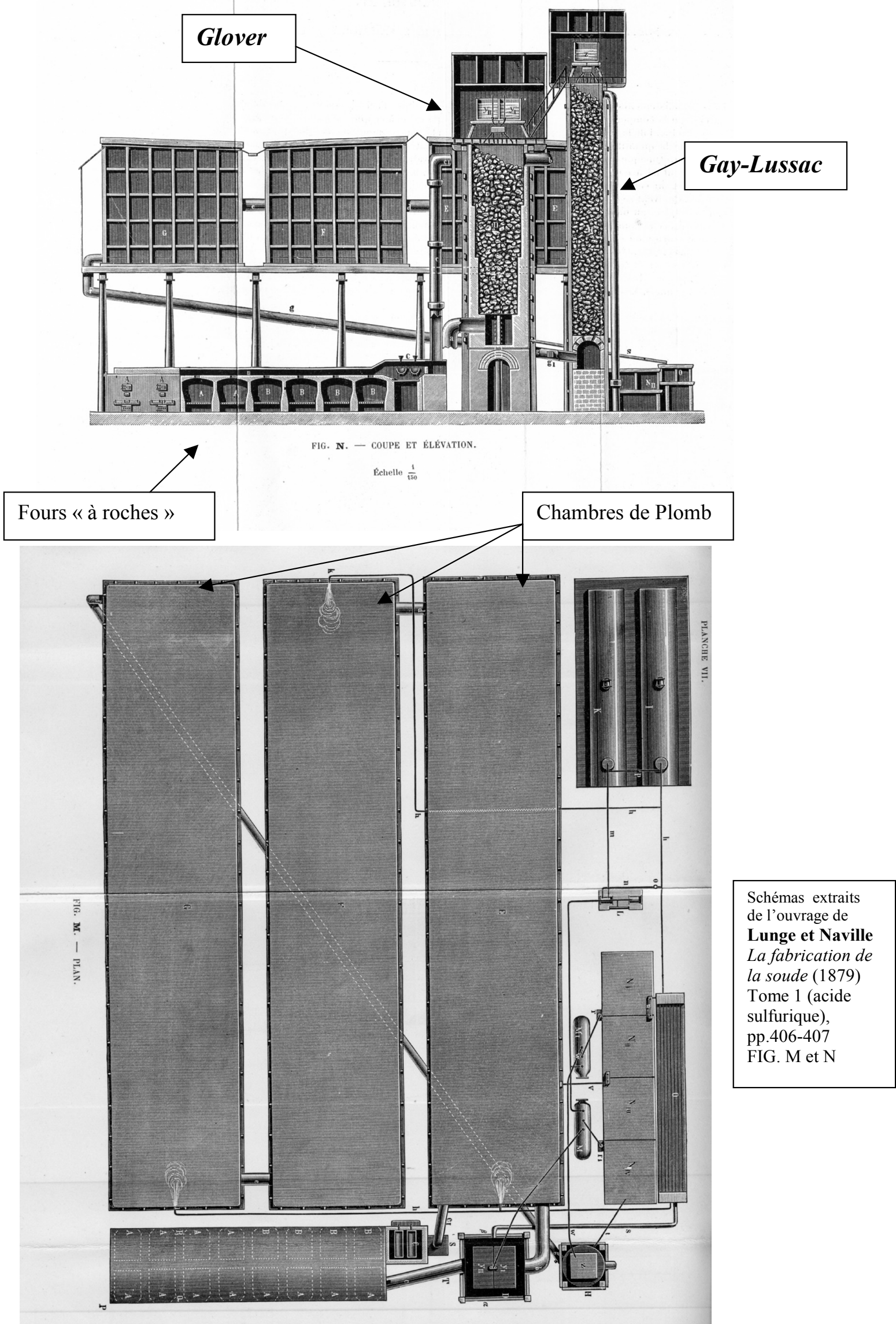
simulation du fonctionnement réel des chambres (sur deux cas d'usines) à l'aide de ce modèle, est finalement assez bien représentative : la décroissance du taux en acide sulfureux au fur et à mesure du passage dans les chambres ne s'éloigne pas trop de la décroissance exponentielle (voir fig. 5 et 6 du texte).

On peut cependant observer une rupture de pente sur la fin, que Sorel explique par une mauvaise maîtrise de la température (p. 364) :

«peu à peu la réaction se ralentit [conformément au modèle cinétique par baisse du taux résiduel en acide sulfureux]. De plus la température ne baisse que très peu par suite de l'incapacité des parois à rayonner toute la chaleur produite dans les régions antérieures et emmagasinée par les gaz [c'est-à-dire un échange thermique insuffisant à la paroi]. Pour ces deux raisons, l'acide de l'intérieur baisse de degré [l'acide formé], la circulation des gaz devient plus lente, leur richesse nitreuse diminue, donc les transports d'oxygène se ralentissent, et la vitesse d'oxydation de l'acide sulfureux devient de plus en plus faible ».

Mais il va un peu plus loin dans son analyse. A l'aide de son modèle, il démontre par exemple que lorsque l'on augmente le débit de manière importante, tout en allongeant dans la même proportion les chambres, cela a pour effet d'augmenter la température des parois en tête et par là, la difficulté de la conduite de I'installation. II écrit, au bas de la p. 364 :

" Imaginons en effet deux appareils ayant même périmètre transversal, et marchant dans des conditions identiques [...] ; mais supposons que l'un soit double de l'autre et reçoive deux fois plus de gaz. Comparons les productions à égale distance de la tête de l'appareil [...]. Le premier dixième de la plus grande chambre produira :

$(a / 100) \cdot(100-58,9)=0,411 a$

Le même volume représentera le cinquième de la première chambre du second appareil. II s'y produira donc :

$(a / 200) \cdot(100-34,6)=0,327 a$

un comportement plus proche d'un autre type de réacteur, à savoir un réacteur où tout serait homogène, appelé selon le vocable du génie des réacteurs, « réacteur continu parfaitement agité ॥. En fait les relevés expérimentaux que mentionne Sorel font état de décroissances linéaires en acide sulfureux qui, même si elles ne sont pas exponentielles, justifient tout à fait la modélisation de Sorel.
[...] Si la température à la paroi du plus petit appareil est $55^{\circ}$, la température extérieure étant de $15^{\circ}[\ldots]$, la température à la paroi du plus grand devra être, à la même distance de l'entrée de [...] $65^{\circ}, 4$. Ainsi, à production égale par mètre cube, une grande chambre sera forcément plus chaude en tête qu'une petite, et la marche sera plus difficile, si on laisse toutes les conditions égales 1 .

Sorel ne donne aucun détail concernant les calculs ci-dessus. Si « a » est probablement assimilable au débit alimenté (a dans un cas, a/2 dans l'autre), les termes entre parenthèses sont à l'évidence les taux en acide sulfureux : $100 \%$ à l'entrée, $58,9 \%$ dans le cas où le débit est le plus fort, $34,6 \%$ dans le cas où le débit est le plus faible. La question est de savoir comment a-t-il déterminé ces valeurs? II a pu en déterminer une des deux à l'aide de son modèle, basé sur une (ou plusieurs) expérience(s) pour le débit en cause. Mais comment a-t-il fait pour l'autre cas, car son modèle ne prend pas en compte apparemment le débit comme paramètre?

Deux possibilités sont à envisager :

- ll a pu avoir des éléments expérimentaux dans les autres conditions de débit, modifier la constante de son modèle et recalculer le nouveaux taux en acide sulfureux?

- Il a su prendre en compte dans son modèle l'effet du débit, sans toutefois le mentionner dans son ouvrage ${ }^{14}$ ?

Avec les connaissances actuelles, l'effet du débit peut être pris en compte, en utilisant la formule du réacteur "piston " précédemment évoquée. "I est intéressant de constater que les valeurs avancées par Sorel sont correctes : si le taux d'acide sulfureux est de $34,6 \%$ pour un débit donné, la valeur pour le débit double est bien de 58,9\% (le calcul donne $58,8 \% !)^{15}$.

14 En l'occurrence c'est bien cette deuxième hypothèse que nous privilégions. II semble en effet vraiment peu probable qu'il ait pu disposer de données mesurées à des débits simples et doubles pour une même "chambre "!

15 Nous avons utilisé pour cela l'équation

(4) : C = Co $\exp \{-k$.V / Qo $\}$, en assimilant les concentrations $C$ aux taux d'acide sulfureux C'est-à-dire $\operatorname{Ln} C / C o=-k$.V / Qo Pour le débit le plus faible, nous avons $\mathrm{Cl}=0,346(\mathrm{Co}=1)$ Ln $0,346=-k . \vee / Q O=-1,061$

Pour un débit double, $2 Q_{0}$, on a donc $-k$. $\vee / 2 Q 0=-0,53$ Donc $C 2=\exp [-0,53]=0,588$, qui est bien la valeur indiquée par Sorel pour un débit double! 
Dans tout ce qui précède, la démarche de Sorel est typique d'une "démarche modélisation " des phénomènes physico-chimiques qu'il étudie (en l'occurrence ici la vitesse de la réaction chimique et les problèmes de transferts thermiques qui lui sont associés), caractéristique du génie des procédés modernes ${ }^{16}$. Toujours dans son ouvrage, on retrouve aussi cette même démarche dans un autre domaine de la fabrication de l'acide sulfurique. Cela concerne cette fois la " tour de Gloven» ${ }^{17}$. Nous ne ferons que citer dans ce cas son approche ${ }^{18}$ :

« Désignons par T la température initiale des gaz, par T1 la température finale, par $\theta$ la température de l'acide à son entrée dans la tour, par $\theta 1$ sa température à la sortie, par t et $\theta$ les température du gaz et de l'acide dans une section distante d'une hauteur h de la grille, par $p$ le produit du poids des gaz par leur chaleur spécifique à pression constante, par P le produit du poids de l'acide par sa chaleur spécifique : la vitesse de refroidissement serait dans l'hypothèse ci-dessus, proportionnelle à la différence des quantités de chaleur apportées par l'acide et par le gaz dans la section considérée : elle serait représentée par :

$-d t / d h=K(p . t-P . \theta)$

[bilan thermique local]

Mais, dans la même hypothèse, on a forcément :

16 Par modélisation, il faut entendre le développement d'un « modèle » mathématique permettant de représenter les phénomènes physico-chimiques en cause, et par là, de prévoir et d'optimiser la mise en œuvre de ces phénomènes. Ces modèles peuvent être de natures différentes, phénoménologiques (basés sur des lois physico-chimiques connues), statistiques, empiriques ou semi-empiriques (on parle parfois de " boites noires ou de "boites grises " à leur propos). Le développement d'un modèle passe toujours par une étape de « validation ॥ à l'aide de résultats connus du phénomène en cause. 17 La « tour de Glover » permet dans la partie haute de libérer les vapeurs nitreuses combinées à l'acide sulfurique par action de l'acide sulfureux alimenté et la vapeur d'eau, et dans la partie basse de réaliser une oxydation directe de l'anhydride sulfureux. La différence de température entre le bas et le haut est très importante : la température est autour de $400^{\circ} \mathrm{C}$ en bas, et voisine de $80-100^{\circ} \mathrm{C}$ en haut. « Le principe de l'appareil consiste à faire couler de haut en bas dans une tour de plomb protégée intérieurement par une enveloppe réfractaire et garnie de matériaux inattaquables, un mélange de l'acide sulfurique nitreux avec la quantité d'acide des chambres, et à faire monter en sens inverse les gaz chauds des fours ") (E. Sorel, la fabrication de l'acide sulfurique, op.cit. note 3, p. 321).

18 E. Sorel, la fabrication de l'acide sulfurique, op.cit. note 3, p. 32.
$\mathrm{P}(\mathrm{T}-\mathrm{t})=\mathrm{P}(\theta 1-\theta)$

[bilan thermique partiel entre le bas et la section $\mathrm{h}$ ]

D'ou : $\quad-d t / d h=K(p . T-P . \theta 1) »$

A partir d'équations de bilans, là encore emblématiques du génie des procédés, Sorel en déduit une relation qui va lui permettre de simuler d'un point de vue théorique la température à l'intérieur du « Glover » et, à travers les écarts observés avec la réalité, d'interpréter les phénomènes en jeu et maîtriser son fonctionnement.

Après la sortie de son livre, Sorel ne poursuit pas ses travaux sur la fabrication de l'acide sulfurique, bien qu'il publie des articles de synthèse sur le sujet jusqu'en 1895'19. Ceci vraisemblablement parce qu'il n'est plus dans le monde industriel (il a démissionné de Saint-Gobain en 1886) et qu'en termes de recherche académique, les aspects "génie des procédés" sont très loin des préoccupations des chimistes de l'époque. II va donc changer complètement de domaine et s'intéresser à la distillation, où il aura une activité particulièrement féconde ${ }^{20}$.

En conclusion, les extraits que nous avons mentionnés relèvent clairement d'un domaine, " le génie des réacteurs " ${ }^{21}$ qui ne verra le jour que près de cinquante ans plus tard à la fin des années 1930. Plus généralement, Sorel apparaît comme un des précurseurs du "génie chimique ", discipline qui n'émerge en France qu'à la fin des années 1940 et qui se généralisera dans les années 1980 à l'ensemble des procédés de transformation de la matière pour devenir le "génie des procédés $\|^{22}$. Sorel continue de mettre en œuvre cette approche de modélisation avec le même succès dans les domaines qu'il aborde par la suite, à savoir la distillation, ef la combustion des moteurs à alcool. La méconnaissance que I'on a de ses apports tient probablement à cette émergence récente en France du génie chimique et à la difficulté de pouvoir identifier des pionniers très en deçà de cette période et en dehors de la communauté des chimistes, dans le cadre très général des « ingénieurs».

\footnotetext{
19 Voir note 5.

20 Voir notes 1 et 2.

21 Voir note 9.

22 Jacques Villermaux, René David, Jean-Léon Houzelot et Daniel Schweich, "Vous avez dit génie des procédés?", L'actualité chimique, mai 1983, pp. 19-27.
} 

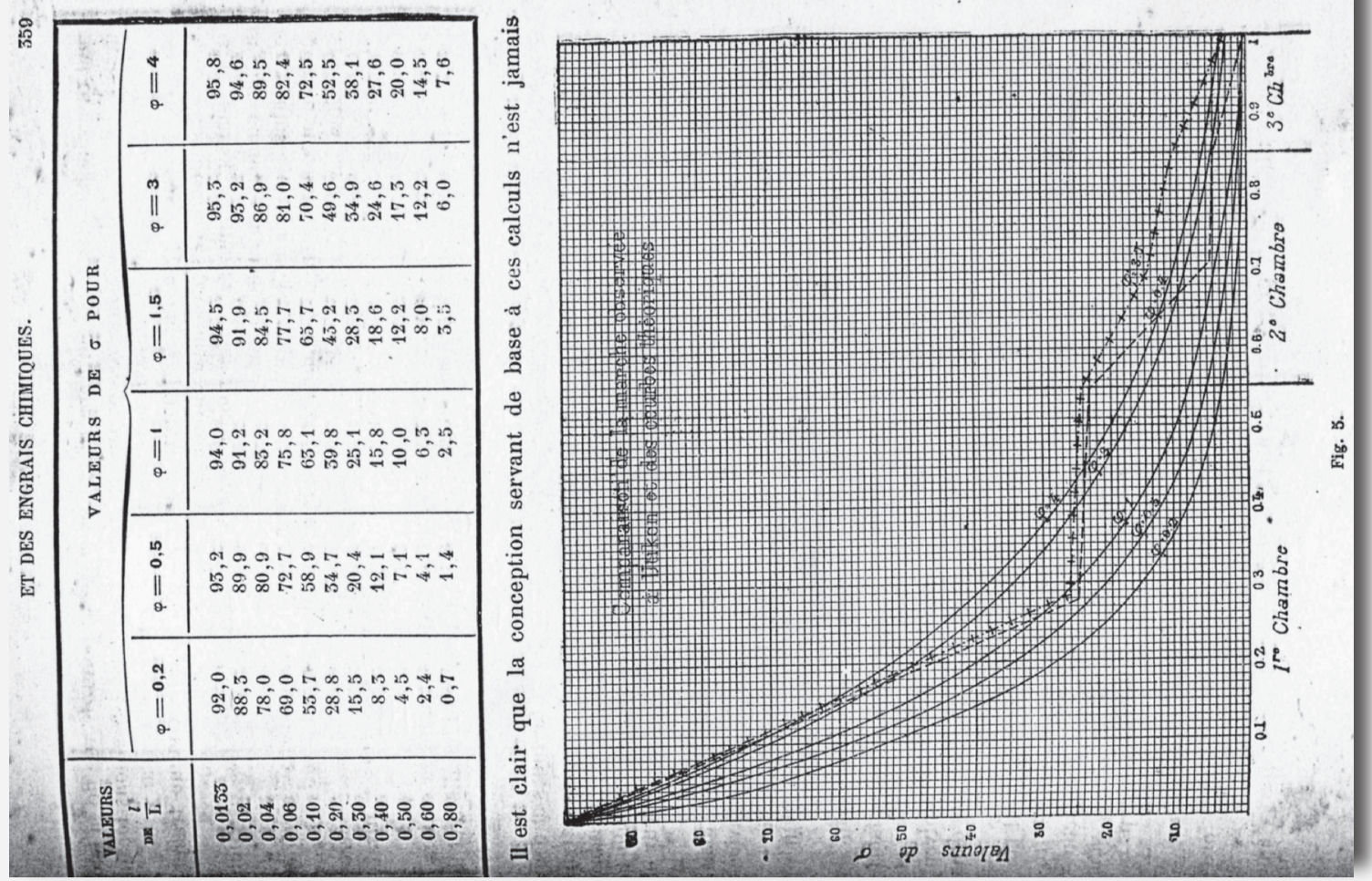

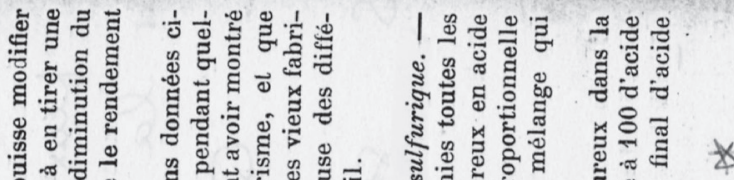

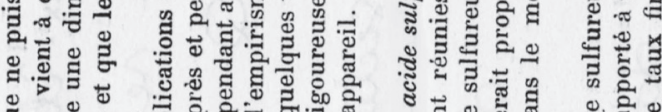

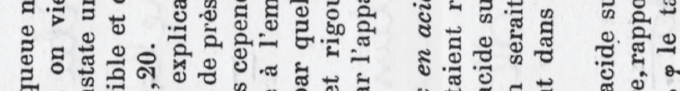

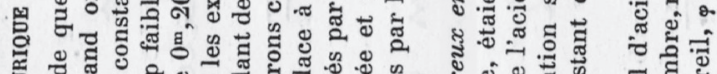

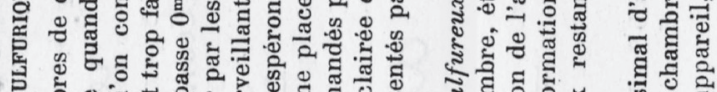

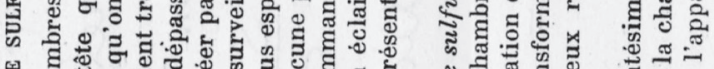

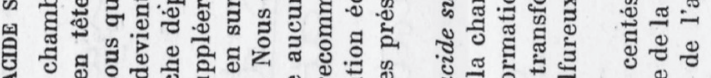

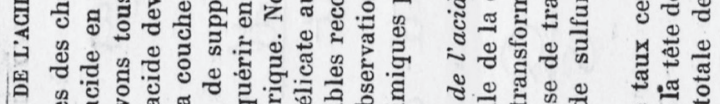

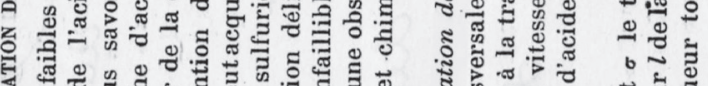

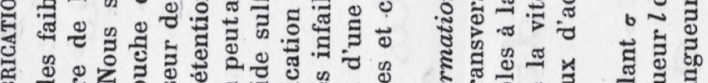

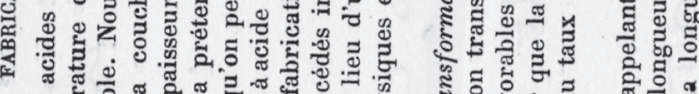
\%

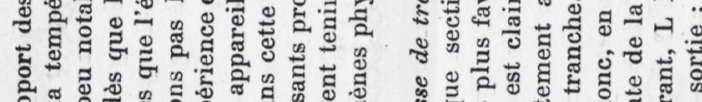

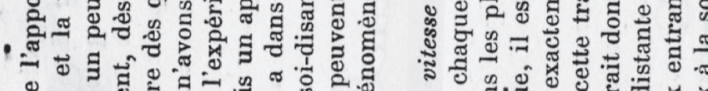
E.

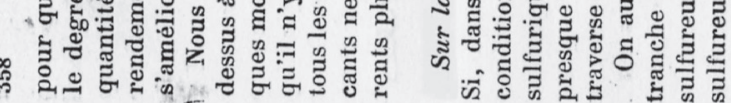

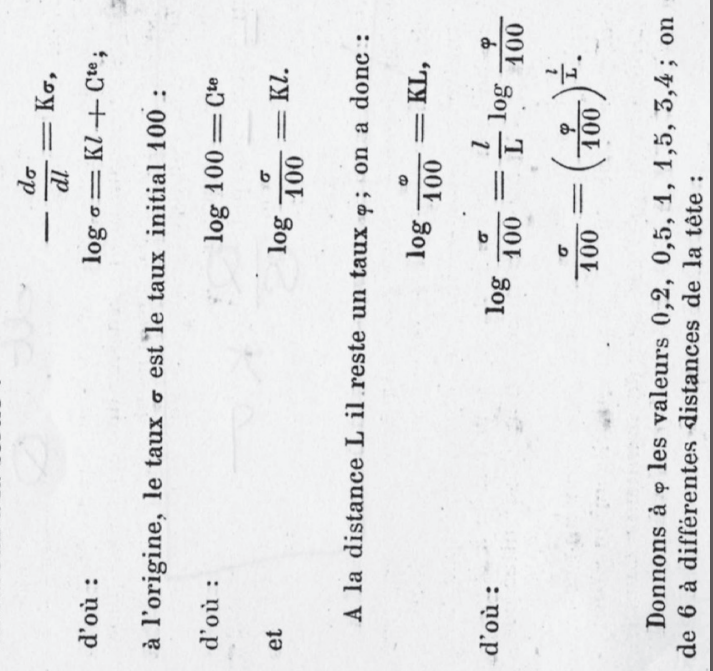



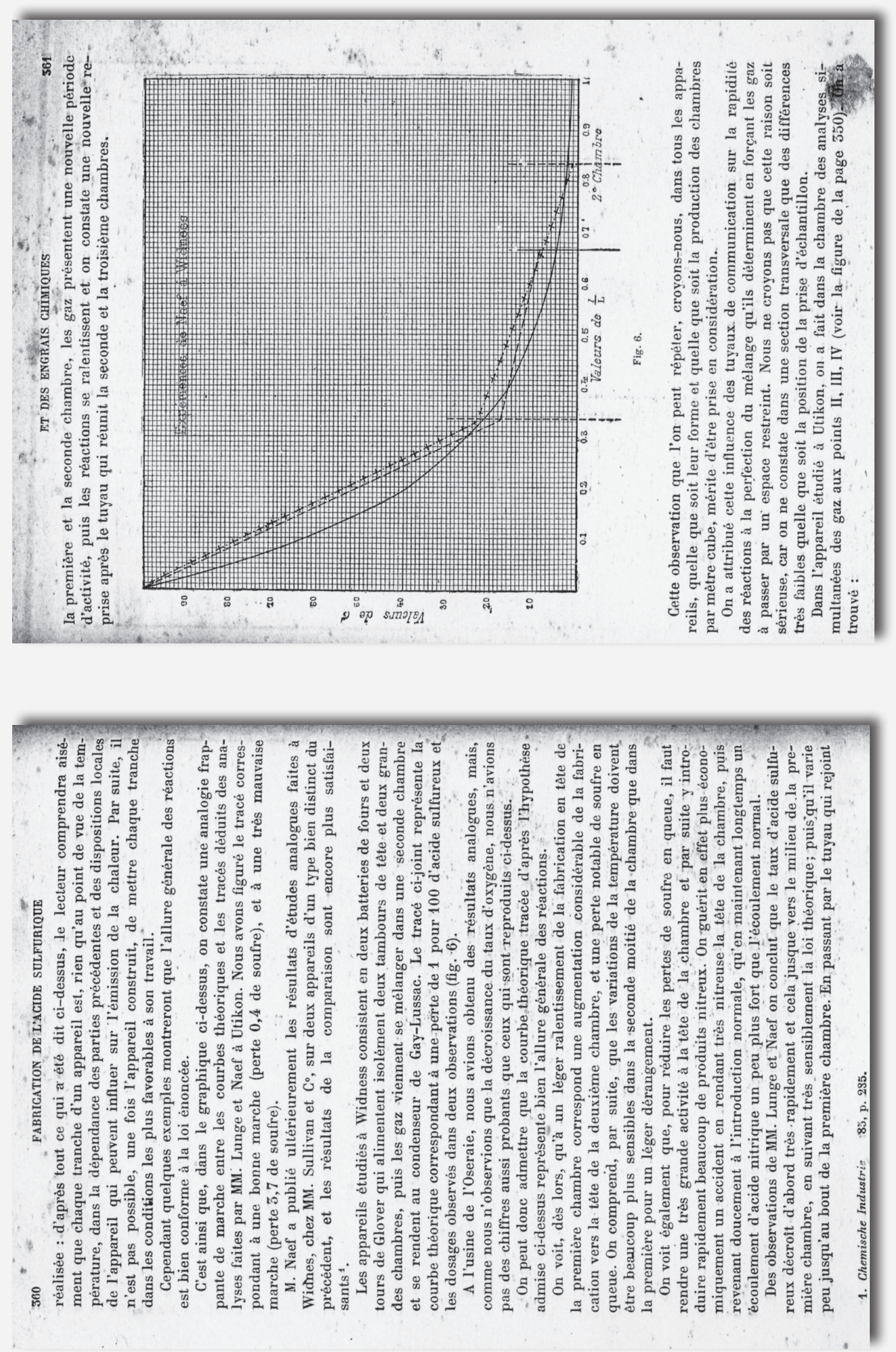

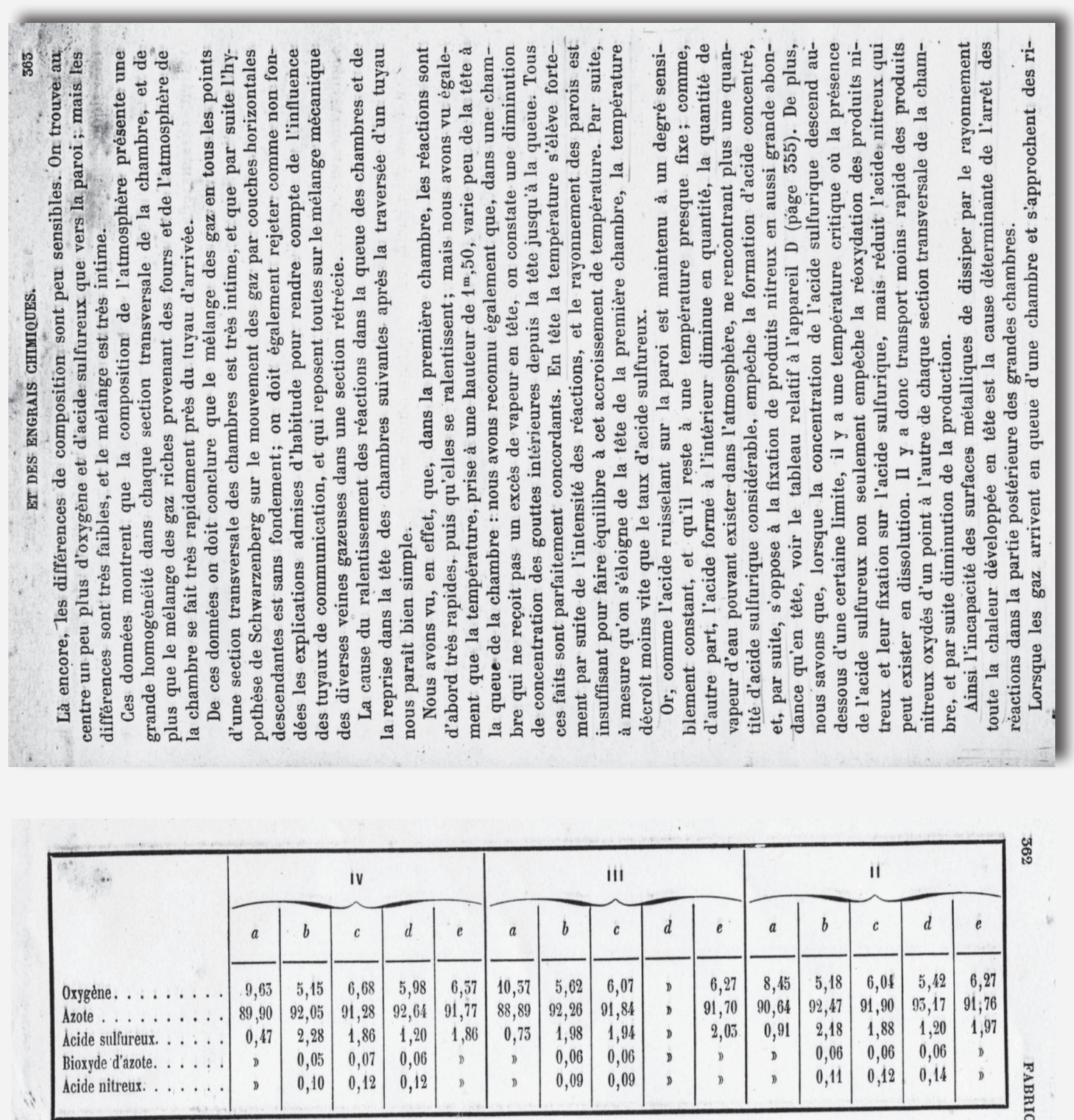

Si l'on tient compte des difficultés de l'analyse, on peut considérer que les différences constatées dans la composition des gaz sont absolument négligeables.

Pour vérifier ce fait, MM. Lunge et Naef ont fait des prises d'échantillons dans l'axe de la clambre en $X$ aux mêmes niveaux que ceux qui correspondent aux points placés vers la paroi et désignés par les chiffres romains II, III, IV. Ils ont observé simultanément dans les six points ainsi déterminés d'une même section transversale :

\begin{tabular}{|c|c|c|c|c|c|c|c|c|c|c|c|c|}
\hline & \multicolumn{4}{|c|}{ HAUT } & \multicolumn{4}{|c|}{ MILIEU } & \multicolumn{4}{|c|}{ BAS } \\
\hline & \multicolumn{2}{|c|}{ PRès de LA PAṚ́̂t } & \multicolumn{2}{|c|}{ a L'INTÉntibur } & \multicolumn{2}{|c|}{ PRÈs DE LA PAROT } & \multicolumn{2}{|c|}{ A L'INTÉrieUn } & \multicolumn{2}{|c|}{ PRÈs DE LA PAROT } & \multicolumn{2}{|c|}{ A L'ITTÉnieur } \\
\hline & $a$ & $b$ & $a$ & $b$ & $a$ & $b$ & $a$ & $b$ & $a$ & $b$ & $a$ & $b$ \\
\hline Oxygène. . . . . . . . . & 7,34 & , & 7,12 & D & 7,76 & $D$ & 7,36 & D & 6,93 & $D$ & 7,39 & D \\
\hline Azote . . . . . . . . & 90,43 & , & 91,07 & ग & 89,98 & $D$ & 90,78 & 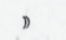 & 90,71 & D & 90,85 & $D$ \\
\hline Acide sulfureux. . . . . . . . & 2,03 & 2,20 & 1,66 & 1,96 & 2,08 & 2,03 & 1,67 & 1,82 & 2,18 & 2,04 & 1,58 & 1,03 \\
\hline Bioxyde d'ázote. . . . . . . & 0,08 & D & 0,08 & D & 0,08 & D & 0,10 & $\gg$ & 0,07 & $D$ & 0,08 & D \\
\hline Acide nitreux $y \%, \ldots$ & 0,12 & D & 0,08 & $D$ & 0,11 & D & 0,09 & D & 0,07 & , & 0,08 & D \\
\hline
\end{tabular}



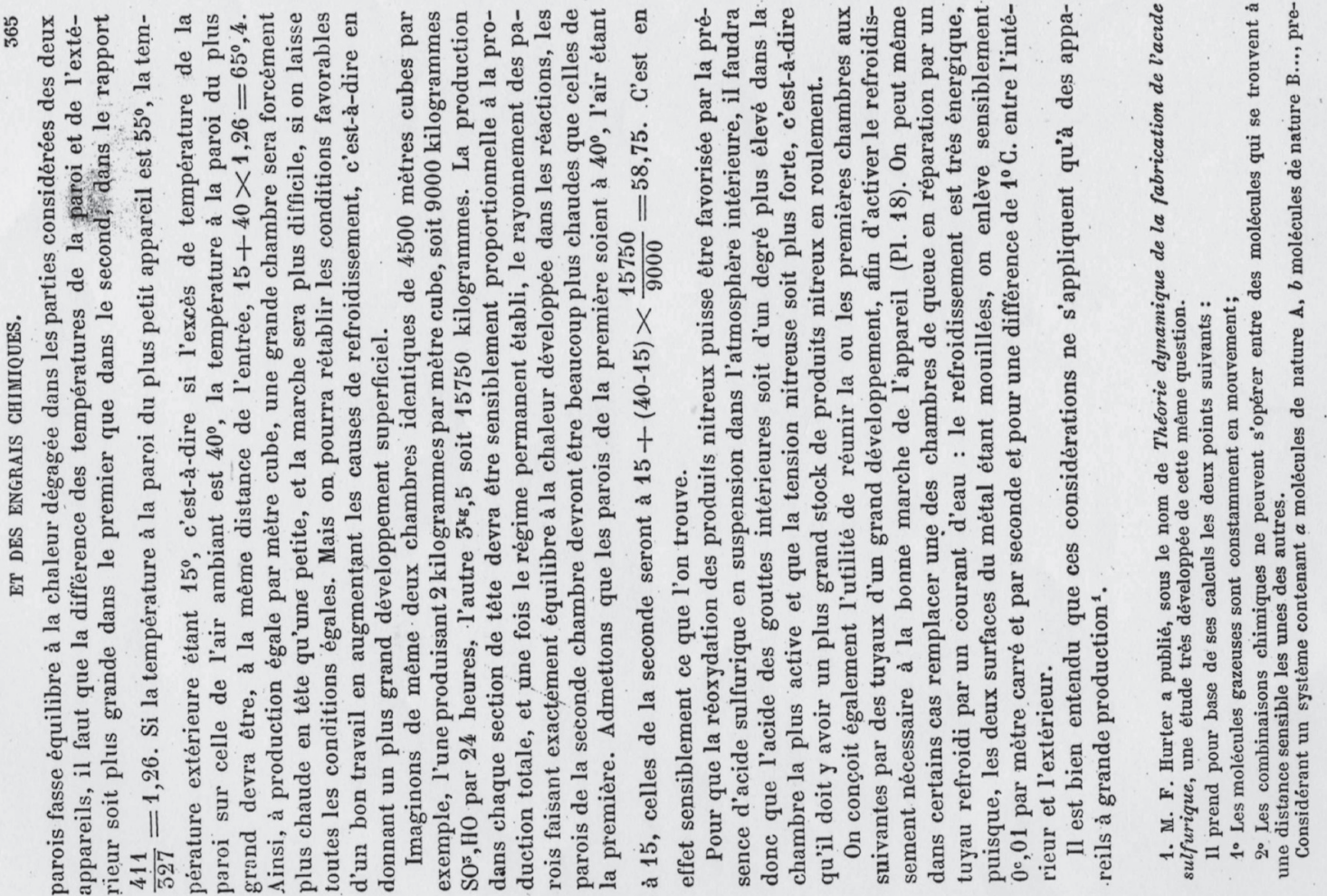

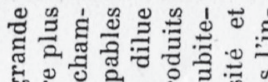

to

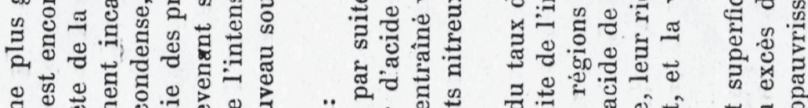

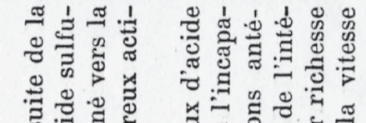

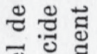

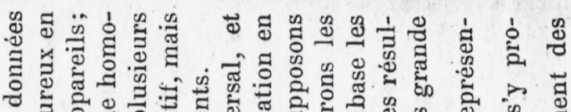

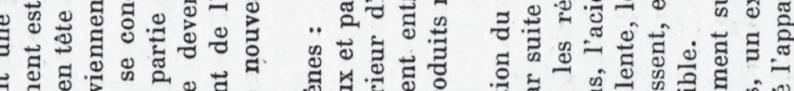

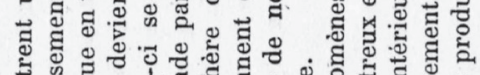

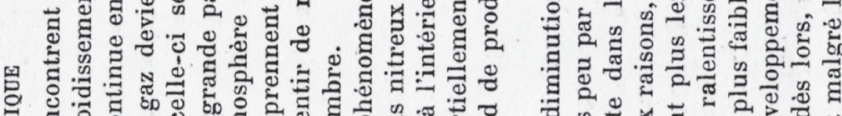

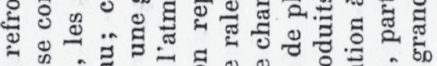

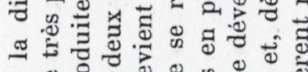

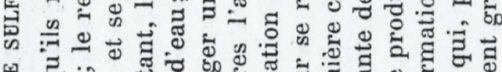

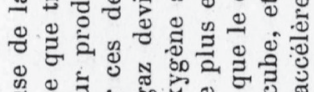

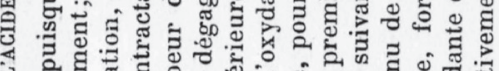

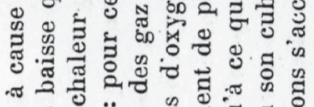

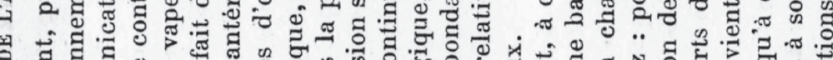

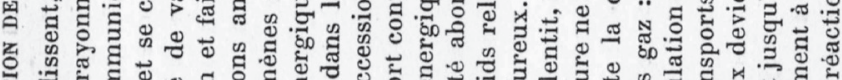

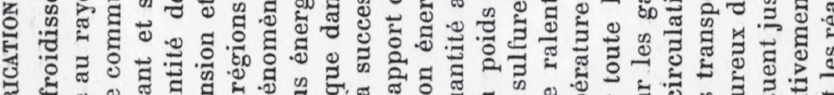

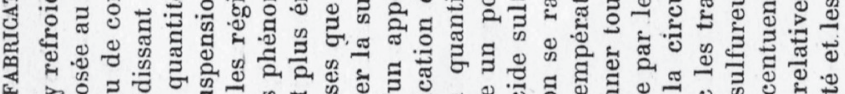

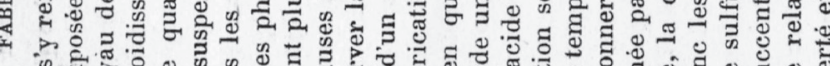
等

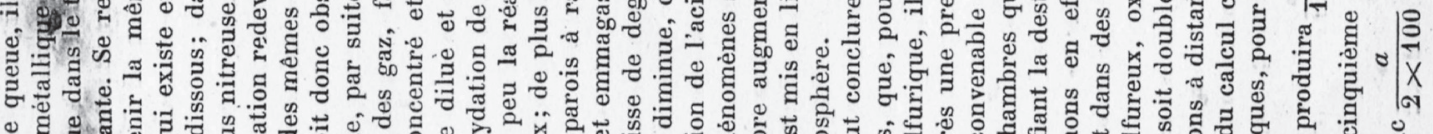
\%

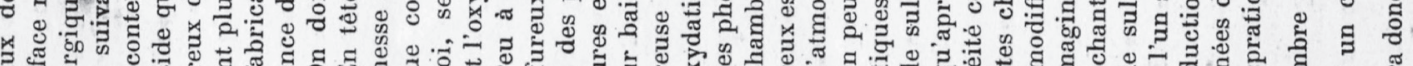

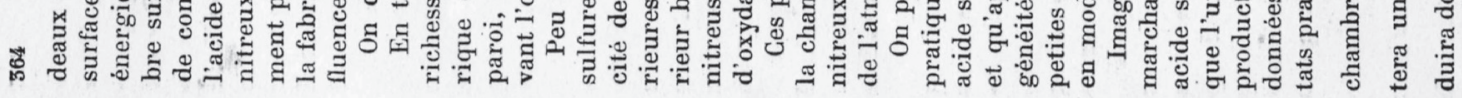

